\title{
CARACTERÍSTICAS FUNCIONAIS PULMONARES E QUALIDADE DE VIDA DE PACIENTE COM INSUFICIÊNCIA CARDÍACA
}

\author{
Pulmonary functional characteristics and quality \\ of life of patients with heart failure
}

\author{
Clarissa Bentes de Araujo Magalhães ${ }^{1 *}$, Patricia Carvalho Bezerra ${ }^{2 * *}$, Thiago Brasileiro de \\ Vasconcelos ${ }^{3^{*}}$, Maria de Jesus Ferreira Marinho ${ }^{4^{*}}$, Andrea da Nóbrega Cirino Nogueira ${ }^{5 * * *}$, \\ Raimunda Hermelinda Maia Macena ${ }^{6^{* * *}}$, Vasco Pinheiro Diógenes Bastos ${ }^{7 * *}$

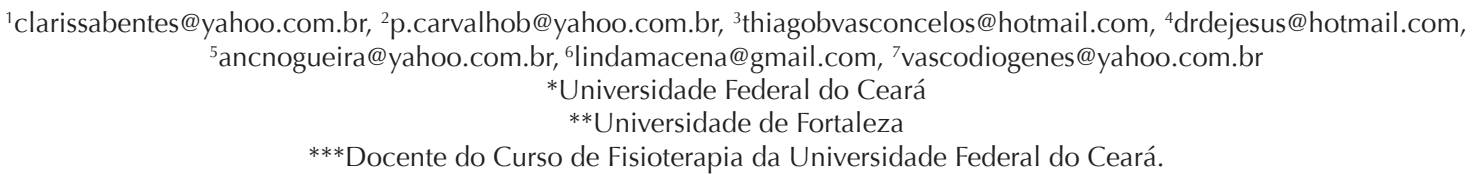

1'clarissabentes@yahoo.com.br, 로.carvalhob@yahoo.com.br, ${ }^{3}$ thiagobvasconcelos@hotmail.com, ${ }^{4}$ drdejesus@hotmail.com, 5ancnogueira@yahoo.com.br, 6 lindamacena@gmail.com, 7vascodiogenes@yahoo.com.br

*Universidade Federal do Ceará

**Universidade de Fortaleza

***Docente do Curso de Fisioterapia da Universidade Federal do Ceará.

Data de entrada do artigo: 02/09/2013

Data de aceite do artigo: 28/04/2014

\section{RESUMO}

Introduçáo: A insuficiência cardíaca (IC) é uma doença crônica em que pacientes apresentam intolerância ao exercício associada aos sintomas de dispneia, fraqueza da musculatura inspiratória e fadiga muscular. Objetivo: Analisar a funçáo pulmonar e qualidade de vida de pacientes com insuficiência cardíaca. Metodologia: Série de casos analisados de maio a dezembro de 2012 no Hospital Universitário Walter Cantídio com 10 indivíduos com IC. Foram incluídos indivíduos com classe funcional II e III, de ambos os sexos, fração de ejeção menor que $45 \%$ e sedentários. Foram avaliadas a função pulmonar através da espirometria e manovacuometria (pressão inspiratória máxima - Pimáx) e a qualidade de vida pelo Short form-36 (SF36) e Minnesota Living with Heart Failure Questionnaire (MLHFQ). Resultado: Dos 10 indivíduos, 70\% eram do sexo masculino, idade média de 51,30 $\pm 10,99$ anos. Quanto à função pulmonar, $100 \%(\mathrm{n}=10)$ apresentaram um padráo obstrutivo e a média da Pimáx foi de $-45,10 \pm 16,15 \mathrm{cmH}_{2} \mathrm{O}$. No SF-36, sete escores estáo abaixo de 50, com comprometimento maior do aspecto físico.A média do MLHFQ foi de 44,50. Conclusão: A funçáo pulmonar e qualidade de vida dos pacientes com IC (classe funcional II e III) estáo comprometidas. Novos estudos devem ser conduzidos envolvendo mais sujeitos para verificar a reprodutibilidade destes achados.

Palavras-chave: Testes de função respiratória; tolerância ao exercício; qualidade de vida; insuficiência cardíac

\section{ABSTRACT}

Introduction: Heart failure (HF) is a chronic disease in which patients present exercise intolerance associated with symptoms of dyspnea, inspiratory muscle weakness and muscle fatigue. Objective: To analyze the pulmonary function and the life quality of patients with heart failure. Methodology: Cases analuzed in the period of May to December 2012 at Hospital Universitário Walter Cantídio with 10 patients diagnosed with HF. We included patients with functional class II and III, of both sexes; ejection fraction less than $45 \%$ and sedentary. We evaluated lung function with spirometry and manovacuometry (Maximum inspiratory pressure - MIP), life quality with the Short Form-36 (SF-36) and Minnesota Living with Heart Failure Questionnaire (MLHFQ). Results: Of the 10 subjects, 70\% were male, mean age $51.30 \pm$ 10.99 years. In relation to lung function, $100 \%(\mathrm{n}=10)$ presented an obstructive pattern and the mean MIP was $-45.10 \pm 16.15 \mathrm{cmH}_{2} \mathrm{O}$. In the SF-36, seven scores are below 50 with greater commitment of the physical aspect. The average MLHFQ was 44.50. Conclusion: Lung function and life quality of patients with HF functional (class II and III) are compromised. Further studies should be conducted with more patients to verify the reproducibility of these findings.

Keywords: Respiratory function tests; exercise tolerance; life quality; heart failure 


\section{Introdução}

A insuficiência cardíaca (IC) é considerada uma doença crônica, resultado da interaçáo de distúrbios hemodinâmicos, neuro-hormonais, imunológicos e metabólicos. As manifestaçóes clínicas da IC originam-se em consequência do débito cardíaco inadequado e/ou acúmulo de sangue em um ou ambos os ventrículos, e geralmente esses pacientes podem queixar-se de dispneia, fadiga muscular, letargia e confusão ${ }^{1}$.

Representa um sério problema de saúde pública. No Brasil, é o motivo de $4 \%$ das internaçóes gerais e $31 \%$ das internaçôes por doenças cardiovasculares ${ }^{2}$. Frequentemente é acompanhada de comorbidades distintas, que interferem no tratamento e na evoluçáo natural da doença, além de dificultar o tratamento e agravar o prognóstico dos portadores ${ }^{3}$.

A intolerância ao exercício é frequentemente associada aos sintomas de dispneia e fadiga muscular, e também pode estar relacionada com o aumento da resposta ventilatória durante o exercício ${ }^{4}$. Os pacientes com IC podem apresentar limitação do débito cardíaco e fraqueza da musculatura inspiratória. Nestas circunstâncias, os músculos inspiratórios e periféricos passam a competir pelo aporte sanguíneo, na tentativa de manter uma ventilação adequada. Diante disso, os músculos ventilatórios desenvolvem fadiga em virtude do baixo débito cardíacos.

A fraqueza da musculatura inspiratória está associada à progressão da $\mathrm{IC}$, principalmente com a diminuição da capacidade de exercício ${ }^{6}$. A disfunção dos músculos ventilatórios é considerada um preditor prognóstico independente ${ }^{7}$.

Dessa forma, é necessário conhecer a função pulmonar e a qualidade de vida de pacientes com IC e assim, com esta análise, poderão ser identificadas as situações de risco, buscar-se soluçóes e o desenvolvimento de açóes precoces para tentar prevenir ou minimizar as limitações apresentadas por estes indivíduos.

Diante disso, o objetivo deste trabalho foi analisar a função pulmonar e a qualidade de vida de pacientes com insuficiência cardíaca.

\section{Metodologia}

Foi feito um estudo descritivo do tipo série de casos desenvolvido no Hospital Universitário Walter Cantídio (HUWC) da Universidade Federal do Ceará, no período de maio a dezembro de 2012. O universo selecionado foram pacientes com diagnóstico clínico de insuficiência cardíaca acompanhados no ambulatório de cardiologia do HUWC, totalizando uma amostra de 10 indivíduos.
Foram incluídos pacientes com insuficiência cardíaca de etiologia variada, classe funcional II e III pela New York Heart Association (NYHA), com idade entre 18 e 70 anos, de ambos os sexos, com fração de ejeção menor que $45 \%$ e sedentários. Foram excluídos pacientes portadores de marca-passo, que apresentassem índice de massa corporal (IMC) maior que 35 $\mathrm{kg} / \mathrm{m}^{2}$, incapacidade de deambulação de origem neurológica ou ortopédica, infarto do miocárdio recente ( $\leq 3$ meses), angina instável, pacientes submetidos à cirurgia cardíaca prévia ( $\leq 3$ meses) e que não assinaram por qualquer razão o termo de consentimento livre e esclarecido. O estudo foi aprovado pelo Comitê de Ética em Pesquisa do HUWC com o protocolo no 038.05.10 e seguiu os preceitos da resoluçáo 196/96 $\mathrm{CNS}^{8}$.

Durante o atendimento ambulatorial rotineiro, os pacientes que preencheram os critérios de inclusão acima foram convidados a participar da pesquisa e assinaram o termo de consentimento livre e esclarecido. Os pacientes foram avaliados em relação à função pulmonar através da espirometria (Spire-Koko ) e manovacuometria digital (Globalmed ${ }^{\ominus}$, modelo MVD 300) e responderam ao questionário de qualidade de vida Short form-36 (SF-36) e Minnesota Living with Heart Failure Questionnaire (MLHFQ).

Para a análise quantitativa dos dados foi utilizado o software estatístico SPSS (versão 20), adotando-se nível de significância de 5\% (p<0,05), de acordo com o teste de normalidade Shapiro-Wilk, e foi utilizada estatística descritiva através da obtenção de distribuiçôes de frequência absolutas e percentuais, bem como média, valor mínimo, máximo, desvio padrão e erro padrão.

\section{Resultados}

Foram estudados 10 indivíduos com IC, sendo 30\% $(\mathrm{n}=3)$ do sexo feminino e $70 \%(\mathrm{n}=7)$ do sexo masculino. A idade variou entre 29 e 63 anos com média de idade de 51,30 $\pm 10,99$ anos ( $p>0,05$; Teste t de Student). Quanto ao IMC, 30\% ( $\mathrm{n}=3)$ estavam com peso normal, $20 \%(n=2)$ com sobrepeso e $50 \%(n=5)$ com obesidade leve.

Os dez pacientes apresentam miocardiopatia dilatada e as causas foram: miocardiopatia dilatada alcoólica em $40 \%(n=4)$, miocardiopatia dilatada idiopática em $20 \%(\mathrm{n}=2)$, miocardiopatia dilatada periparto em $10 \%$ $(\mathrm{n}=1)$ e $30 \%(\mathrm{n}=3)$ com miocardiopatia dilatada a esclarecer. Em relação à classe funcional de NYHA, 30\% $(n=3)$ encontravam-se na classe II e $70 \%(n=7)$ na classe III. A fração de ejeção variou de $26 \%$ a $42 \%$, com média de 35,60 \pm 5,66\% $(p>0,05$; Teste $t$ de Student $)$. 
Todos os pacientes eram sedentários e nenhum apresentou pneumopatia prévia.

A tabela 1 demonstra os valores obtidos de algumas variáveis espirométricas, como capacidade vital forçada (CVF), volume expiratório forçado no $1^{\circ}$ segundo (VEF1), volume expiratório forçado no $1^{\circ}$ segundo/ capacidade vital forçada (VEF1/CVF) dos pacientes portadores de IC. Observa-se que 100\% dos indivíduos apresentaram um padrão obstrutivo.

Tabela 1: Distribuição dos dados de acordo com os valores da prova de função pulmonar. Fortaleza/CE, 2012

\begin{tabular}{lccc}
\hline $\begin{array}{l}\text { Variáveis } \\
\text { espirométricas }\end{array}$ & $\begin{array}{c}\text { Média do } \\
\text { valor predito } \\
\text { (Erro padráo) }\end{array}$ & $\begin{array}{c}\text { Valor obtido } \\
\text { (Erro padrão) }\end{array}$ & $\begin{array}{c}\% \text { predito } \\
\text { (Erro padráo) }\end{array}$ \\
\hline VCF & $3,52( \pm 0,23)$ & $2,89( \pm 0,28)$ & $82,72( \pm 6,57)$ \\
VEF1 & $2,97( \pm 0,12)$ & $2,08( \pm 0,16)$ & $72,68( \pm 6,21)$ \\
VEF1/CVF & $87,85( \pm 7,58)$ & $73,20( \pm 3,36)$ & $83,12( \pm 3,39)$ \\
\hline
\end{tabular}

A análise da pressão inspiratória máxima (Pimáx) obtida pelo manovacuômetro digital demonstrou que $100 \%$ da amostra apresentam reduçáo da Pimáx. A Pimáx variou de $-17 \mathrm{cmH}_{2} \mathrm{O}$ a $-68 \mathrm{cmH}_{2} \mathrm{O}$ com média de $-45,10 \pm 5,10 \mathrm{cmH}_{2} \mathrm{O}$ (p $<0,05$; Teste t de Student) (Gráfico 1).

Figura 1: Pressão inspiratória máxima dos pacientes com diagnóstico clínico de insuficiência cardíaca.

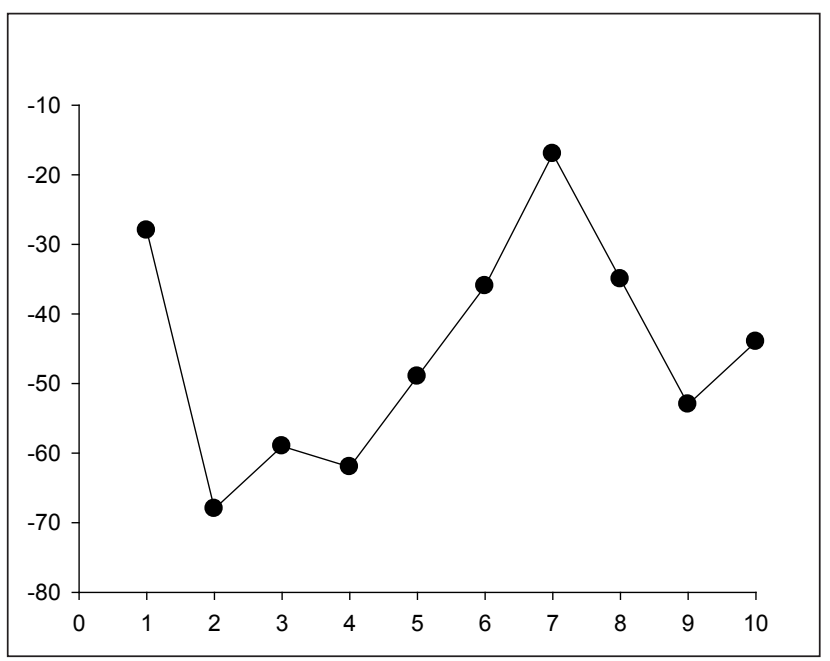

Os dados referentes à pontuação dos pacientes de acordo com o SF-36 e o questionário de Minnesota estão descritos na tabela 2. Assim, observa-se que, das oito dimensôes de QV avaliadas, em sete foram identificados escores abaixo de 50 . Em relação ao questionário de Minnesota a média foi de 44,50.
Tabela 2: Pontuação do SF-36 e questionário de Minnesota de pacientes com diagnóstico clínico de insuficiência cardíaca

\begin{tabular}{lcccc}
\hline \multicolumn{1}{c}{ Dimensóes } & Média & $\begin{array}{c}\text { Valor } \\
\text { mínino }\end{array}$ & $\begin{array}{c}\text { Valor } \\
\text { máximo }\end{array}$ & $\begin{array}{c}\text { Erro } \\
\text { padrão }\end{array}$ \\
\hline SF-36 & & & & \\
$\quad$ Saúde mental (SM) & 38,40 & 16 & 72 & 6,17 \\
Vitalidade (V) & 43,50 & 15 & 70 & 4,59 \\
Aspectos sociais (AS) & 56,20 & 38 & 88 & 4,26 \\
Dor & 44,20 & 32 & 68 & 3,56 \\
Estado Geral de & 41,00 & 25 & 70 & 4,98 \\
Saúde (EGS) & & & & \\
Capacidade & 28,10 & 11 & 39 & 2,67 \\
$\quad$ Funcional (CF) & & & & \\
$\quad$ Aspectos & 6,60 & 0 & 33 & 4,40 \\
$\quad$ Emocionais (AE) & & & & \\
$\quad$ Aspectos físicos (AF) & 5,00 & 0 & 25 & 3,33 \\
Minnesota & 44,50 & 19 & 76 & 6,61 \\
\hline
\end{tabular}

Saúde mental $=$ SM; Vitalidade $=$ V; Aspecto social $=$ AS; Estado Geral de Saúde = EGS; Capacidade Funcional = CF; Aspectos Emocionais = AE; Aspectos físicos = AF.

\section{Discussão}

O músculo diafragma está envolvido na maior parte do trabalho ventilatório e $55 \%$ de suas fibras são do tipo 1. Este aspecto fisiológico faz que o diafragma tenha um maior fluxo sanguíneo por grama de tecido do que qualquer outro músculo esquelético do corpo. A redução da fração de ejeção, encontrada em pacientes com IC, interfere no fluxo sanguíneo para o diafragma levando a uma alteração na sua função .

Vários estudos demonstram que a força do músculo inspiratório está frequentemente diminuída em pacientes com IC, e isso é possivelmente desencadeado pela redução da fração de ejeção, favorecendo a deteriorização de seu estado cardíaco funcional ${ }^{10-14}$. Corroborando nossos resultados, Harrington e Coast ${ }^{10}$ destacaram que a fraqueza muscular pode resultar em dispneia devido à redução da pressão inspiratória e da expiratória máxima observadas nestes pacientes.

No estudo de Meyer et al. ${ }^{11}$, que avaliou a pressão muscular ventilatória em 244 indivíduos com IC, observou-se uma redução da Pimáx em 100\% dos pacientes, dados estes confirmados pelo nosso estudo. Já no estudo de Hughes et al. ${ }^{12}$ houve uma diminuição de $28 \%$ da Pimáx. Evans et al. ${ }^{13}$ também encontraram em seu estudo uma redução da força muscular respiratória.

$\mathrm{O}$ estudo de Forgiarini-Junior et al. ${ }^{14}$ avaliou a força muscular respiratória e função pulmonar em pacientes com IC, e encontrou que há um decréscimo da função pulmonar e da força muscular respiratória nos pacientes em classe funcional III em relação à classe funcional II. 
A força muscular respiratória alterada em pacientes com IC demonstra que existe diminuição da função pulmonar, em consequência da redução da força muscular respiratória ${ }^{14}$. Em indivíduos com IC, podemos evidenciar a presença de um padráo ventilatório do tipo restritivo e/ou obstrutivo, pois ocorre alteraçóes na função ventilatória, incluindo os volumes pulmonares e a função muscular respiratória ${ }^{15}$. Uma redução da capacidade vital é registrada nesses pacientes ${ }^{16}$.

Em estudo realizado com pacientes portadores de IC em todas as classes funcionais (I, II, III e IV) da NYHA, foi observada a diminuição significativa dos volumes pulmonares, especialmente na CVF e no VEF $1^{17}$. Neste estudo encontramos que $100 \%$ dos pacientes apresentaram padrâo ventilatório obstrutivo.

O padrão ventilatório obstrutivo verificado em alguns pacientes com IC pode ser uma consequência da congestáo das veias pulmonares e do edema intersticial. Isso aumenta a resistência das vias aéreas e causa colapso expiratório prematuro das vias aéreas distais, o que gera aprisionamento de $\mathrm{ar}^{6}$. Outro ponto a ser abordado é a presença de um IMC caracterizando obesidade. Este fato confirma um maior comprometimento da função respiratória devido ao aumento de massa na regiáo torácica e abdominal ${ }^{16}$. Neste estudo, 2 pacientes apresentaram sobrepeso e 4 obesidade leve, fatores que podem ser agravantes do compromentimento da função respiratória.

A maioria dos estudos que envolve a avaliação da QV emprega questionários genéricos. Dessa forma, empregamos, além do questionário genérico SF-36, o questionário específico MLHFQ, sendo o mais utilizado para avaliação do impacto da IC diante da $\mathrm{QV}^{18-19}$.

No presente estudo, a QV medida pelo SF-36 encontrou-se alterada em quase todos os domínios (AF, $\mathrm{AE}, \mathrm{CF}$, Dor, EGS, V e SM), mas especialmente mostrou comprometimento maior no aspecto físico, que avalia como a saúde física interfere nas atividades do trabalho - o que demonstra, portanto, que a limitação funcional imposta pela doença está associada à piora da QV. Nossos achados são semelhantes àqueles evidenciados no estudo de Sneed et al. ${ }^{20}$, que revelaram escores baixos para todos os domínios do SF-36, especialmente quanto ao aspecto físico de pacientes com IC.

Evidenciamos um escore total elevado para o MLHFQ $(44,50)$, o que implica piora da QV da nossa amostra. Estudos recentes concluíram que a variação do escore total do MLHFQ entre 27,7 e 42,7 apresenta associação com a classe funcional II e III proposta pela NYHA, respectivamente ${ }^{18,21}$. Esse fato comprova nossos dados, no qual a classe funcional (NYHA) variou entre II a III.

Uma das limitações do estudo foi o número de participantes da pesquisa; seriam necessários mais voluntários para obtermos uma melhor observação do comportamento das variáveis analisadas e da repercussão na qualidade de vida dos pacientes com IC.

\section{Conclusão}

A função pulmonar e a qualidade de vida estão comprometidas em pacientes com IC classe II e III. O aspecto físico da qualidade de vida é o mais atingido.

Com o conhecimento das possíveis complicaçóes que os pacientes com IC podem apresentar, a busca por ações precoces, como um programa de reabilitação cardíaca ou treinamento muscular inspiratório, pode ser uma importante contribuição para tentar prevenir ou minimizar as limitações apresentadas por estes indivíduos, e assim melhorar seus sintomas, prognóstico e qualidade de vida. Novos estudos envolvendo maior número de indivíduos devem ser realizados para verificar a reprodutibilidade destes achados.

\section{Referências}

1. Barnes TA. Core textbook of respiratory care practice. $2^{\mathrm{a}}$ ed. Missouri: Mosby Inc.; 1994.

2. Hunt SA, Abraham WT, Chin MH, Feldman AM, Francis GS, Ganiats TG et al. ACC/AHA 2005 guideline update for the diagnosis and management of chronic heart failure in the adult: a report of the American College of Cardiology/American Heart Association Task Force on Practice Guidelines (Writing Committee to Update the 2001 Guidelines for the Evaluation and Management of Heart Failure). Circulation. 2005; 112(12):154-235.

3. Stewart S, Jenkins A, Buchan S, McGuire A, Capewell S, McMurray JJ. The current cost of heart failure to the National Health Service in the UK. Eur J Heart Fail. 2002; 4(3):361-71.

4. Cotter G, Moskovitz Y, Milovanov O, Salah A, Blatt A, Krakover $\mathrm{R}$ et al. Acute heart failure: a novel approach to its pathogenesis and treatment. Eur J Heart Fail. 2002; 4(3):227-34.

5. Powers SK, Coombes J, Demirel H. Exercise training-induced changes in respiratory muscles. Sports Med. 1997; 24(2):120-31.

6. Meyer FJ, Zugck C, Haass M, Otterspoor L, Strasser RH, Kübler $\mathrm{W}$ et al. Inefficient ventilation and reduced respiratory muscle capacity in congestive heart failure. Basic Res Cardiol. 2000; 95(4):333-42.

7. Dall'ago P, Chiappa GR, Guths H, Stein R, Ribeiro JP. Inspiratory muscle training in patients with heart failure and inspiratory muscle weakness: a randomized trial. J Am Coll Cardiol. 2006; 47(4):757-63. 
8. Brasil. Resolução Conselho Nacional de Saúde, n. ${ }^{\circ}$ 196, de 10 de outubro de 1996. Aprova diretrizes e normas regulamentadoras de pesquisa envolvendo seres humanos. Diário Oficial da União. 1996; 201(Seção 1). p. 21082.

9. Hammond MD, Bauer KA, Sharp JT, Rocha RD. Respiratory muscle strength in congestive heart failure in congestive heart failure. Chest. 1990; 98(5):1091-4.

10. Harrington D, Coast AJS. Skeletal muscle abnormalities and evidence for their role in symptom generation in chronic heart failure. Eur Heart J. 1997; 18(12):1865-72.

11. Meyer FJ, Mathias MB, Zugck C, Kirschke A, Scellberg D et al. Respiratory muscle dysfunction in congestive heart failure. Circulation. 2001; 103:2153-2158.

12. Hughes PD, Polkey MI, Lou Harris M, Coats AJS, Moxham J, Green M. Diaphragm strength in chronic heart failure. Am J Respir Crit Care Med. 1999; 160(2):529-34.

13. Evans AS, Watson L, Hawkins M, Cowley AJ, Johnston DA, Kinnear WJM. Respiratory muscle strenght in chronic heart failure. Thorax. 1995; 50(6):625-28.

14. Forgiarini-Junior LA, Rubleski A, Garcia D, Tieppo J, Vercelino R, Dal Bosco A et al. Avaliação da força muscular respiratória e da função pulmonar em pacientes com insuficiência cardíaca. Arq Bras Cardiol. 2007; 89(1):36-41.

15. Witt C, Borges AC, Haake H, Reindl I, Kleber FX, Baumann G. Respiratory muscle weakness and normal ventilator drive in dilative cardiomyopathy. Eur Heart J. 1997; 18(8):1322-8.

16. Gonzaga FMG. Função pulmonar em pacientes portadores de insuficiência cardíaca congestiva associada a distúrbios respiratórios do sono [tese]. São José dos Campos: Universidade do Vale do Paraíba; 2006.

17. Johnson B, Olson LJ, O’Malley KA, Allison TG, Squires RW. Pulmonary function in patients with reduced left ventricular function. Chest. 2001; 120(6):1869-76.

18. Nogueira IDB, Servantes DM, Nogueira PAMS, Pelcerman A, Salvetti XM, Salles F et al. Correlação entre qualidade de vida e capacidade funcional na insuficiência cardíaca. Arq Bras Cardiol. 2010; 95(2):238-243.

19. Riegel B, Moser DK, Glaser D, Carlson B, Deaton C, Armola $\mathrm{R}$ et al. The Minnesota living with heart failure questionnaire: sensitivity to differences and responsiveness to intervention intensity in a clinical population. Nurs Res. 2002; 51(4):209-18.

20. Sneed NV, Paul S, Michel Y, VanBakel A, Hendrix G. Evaluation of 3 quality of life measurement tools in patients with chronic heart failure. Heart Lung. 2001; 30(5):332-40.

21. Meyer K, Laederach-Hofmann K. Effects of a comprehensive rehabilitation program on quality of life in patients with chronic heart failure. Prog Cardiovasc Nurs. 2003; 18(4):169-76. 